Article

\title{
Probiotic Cocktail Identified by Microbial Network Analysis Inhibits Growth, Virulence Gene Expression, and Host Cell Colonization of Vancomycin-Resistant Enterococci
}

\author{
Wei-Sheng Sun ${ }^{1,2}$, Yuarn-Jang Lee ${ }^{3,4}$, Kun-Nan Tsai ${ }^{5}$, Yu-Hsuan $\mathrm{Ho}^{5}$ and \\ Shiuh-Bin Fang $1,2,6, *($ ) \\ 1 Division of Pediatric Gastroenterology and Hepatology, Department of Pediatrics, Shuang Ho Hospital, \\ Taipei Medical University, Taipei 23561, Taiwan; adun1943@gmail.com \\ 2 Department of Pediatrics, School of Medicine, College of Medicine, Taipei Medical University, Taipei 110301, \\ Taiwan \\ 3 Division of Infectious Disease, Department of Internal Medicine, Taipei Medical University Hospital, \\ Taipei 110301, Taiwan; yuarn@tmuh.org.tw \\ 4 Department of Internal Medicine, School of Medicine, College of Medicine, Taipei Medical University, \\ Taipei 110301, Taiwan \\ 5 Delta Research Center, Delta Electronics, Inc., Taipei 114501, Taiwan; brian.kn.tsai@deltaww.com (K.-N.T.); \\ kenseifan@gmail.com (Y.-H.H.) \\ 6 Master Program for Clinical Pharmacogenomics and Pharmacoproteomics, College of Pharmacy, \\ Taipei Medical University, Taipei 110301, Taiwan \\ * Correspondence: sbfang@tmu.edu.tw; Tel.: +886-2-2249-0088 (ext. 2951)
}

Received: 25 April 2020; Accepted: 27 May 2020; Published: 29 May 2020

\begin{abstract}
The prevalence of vancomycin resistant enterococcus (VRE) carrier-state has been increasing in patients of intensive care unit and it would be a public health threat. Different research groups conducted decolonizing VRE with probiotic and the results were controversial. Therefore, a systemic approach to search for the probiotic species capable of decolonizing VRE is necessary. Thus, VRE was co-cultured with ten probiotic species. The fluctuations of each bacterial population were analyzed by $16 \mathrm{~S}$ rRNA sequencing. Microbial network analysis (MNA) was exploited to identify the most critical species in inhibiting the VRE population. The MNA-selected probiotic cocktail was then validated for its efficacy in inhibiting VRE, decolonizing VRE from Caco-2 cells via three approaches: exclusion, competition, and displacement. Finally, the expression of VRE virulence genes after co-incubation with the probiotic cocktail were analyzed with quantitative real-time PCR (qRT-PCR). The MNA-selected probiotic cocktail includes Bacillus coagulans, Lactobacillus rhamnosus GG, Lactobacillus reuteri, and Lactobacillus acidophilus. This probiotic combination significantly reduces the population of co-cultured VRE and prevents VRE from binding to Caco-2 cells by down-regulating several host-adhesion genes of VRE. Our results suggested the potential of this four-strain probiotic cocktail in clinical application for the decolonization of VRE in human gut.
\end{abstract}

Keywords: probiotics; microbial network analysis; colonization; vancomycin-resistant enterococci; $16 \mathrm{~S}$ rRNA sequencing

\section{Introduction}

Enterococci, which are harmless commensal bacteria in the human gut [1], can cause opportunistic infections in immunocompromised individuals [2]. Enterococci acquire mobile genetic elements and develop resistance to multiple antibiotics, including the last-resort drugs vancomycin and linezolid [3-5]. 
Hospital-acquired infections that are caused by antibiotic-resistant Enterococcus species are difficult to treat and they can be life threatening; consequently, they have become a major public health concern and increased the economic burden of patients and governments [6-8]. Furthermore, Enterococcus species may transfer antibiotic resistance genes through conjugative plasmids to other clinically hazardous microbes [4], e.g., gram-positive Staphylococcus aureus [9] and gram-negative Escherichia coli [10]. Among hospital-acquired infections that are caused by Enterococcus species, E. faecalis and E. faecium account for the majority of infections, surpassing other Enterococcus species, including E. casseliflavus, E. gallinarum, E.durans, E. avium, E. hirae, E. raffinosus, and E. mundtii [11-14]. In 2017, the WHO regarded vancomycin-resistant enterococci (VRE) as requiring high priority for research and development of new antibiotics, owing to their global prevalence and the aforementioned risks to public health [15]. In addition to being the most relevant to hospital-acquired infections, E. faecalis and E. faecium are also the two main reservoirs for $v a n A, \operatorname{van} B$, and van $M$, which are the genes contributing to high levels of resistance to vancomycin; therefore, they are the main targets of studies focused on VRE. Vancomycin-resistant E. faecalis has been more frequently detected than other Enterococcus species; however, E. faecium is recently being increasingly detected [16,17].

Studies have indicated that probiotics might improve human health by producing metabolites that provide nutrition to human body and competing with pathogenic microorganisms [18]. To fight against pathogens, probiotic uptake provides indirect protection through immunomodulation and enhancement of the intestinal epithelial barrier [19-21] and direct protection through the secretion of antimicrobial molecules [22,23]; hence, the potential of probiotics in fighting against antibiotic-resistant pathogenic bacteria warrants more evaluation. Lactobacillus species inhibit pathogenic bacteria by producing short-chain fatty acids, hydrogen peroxide, and bacteriocins [24]. Several in vitro studies have indicated that Lactobacillus rhamnosus GG (LGG) and L. reuteri can antagonize VRE by reducing the attachment and colonization of VRE $[25,26]$. Inspired by in vitro studies, clinical trials aiming to investigate the potential of decolonizing VRE in the human gut were conducted. However, many studies did not include a reasonable cohort size, and the results were controversial. Szachta et al. reported that administering LGG to pediatric VRE carriers significantly reduced their VRE carrier status when compared with children in the placebo group [27]. However, administering LGG to adult participants with VRE carrier status in another study that was conducted by Doron et al. did not significantly reduce VRE colonization [28]. Therefore, approaches to find appropriate probiotic strains for decolonizing VRE colonization requires more studies for clarification. Studies comparing the efficacies of probiotics in a mixture and their separate strains have suggested the advantage of the probiotic mixture in improving the modulation of gut microbiota and eradicating Helicobacter pylori [29]. Hence, the application of probiotic mixtures to decolonize VRE in the human gut is a worthwhile attempt.

We included several probiotic species and investigated their potential of inhibiting VRE in an in vitro co-culture model to identify an effective probiotic mixture. In this study, rather than random selection of probiotic bacteria, we used a systemic approach, the microbial network analysis (MNA), which used the rule-based microbial network (RMN) algorithm [30] in order to analyze cooperative and competitive interactions among hundreds of bacterial species and infer the directions of these interactions. Thus, the optimal combination of probiotic strains for inhibiting VRE was identified. The efficacy of such a probiotic cocktail was compared with those of individual strains in the selected combination. In this study, we adopted in vitro methods, including co-culture, exclusion assay, competition assay, displacement assay, and the quantification of VRE virulence gene expression, in order to prove the potential of the probiotic cocktail that was selected by MNA in repressing the population and virulence gene expression of VRE. 


\section{Materials and Methods}

\subsection{Bacterial Strains and Culture}

Table S1 lists bacterial strains, their sources, and growth conditions used in this study. VRE strains used were the clinical isolates of vancomycin-resistant E. faecium (VREfm) from Taipei Medical University Hospital (TMUH) and vancomycin-resistant E. faecalis (VREfs) from Taipei Medical University-Shang Ho Hospital (Table S1). Bacillus coagulans (ATCC 7050/BCRC 10606), Bifidobacterium bifidum (ATCC 15696/BCRC 14614), B. longum subsp. infantis (ATCC 15697/BCRC 15416), Lactobacillus rhamnosus GG (ATCC 53103/BCRC 16000), Lactococcus lactis subsp. lactis (CSCC 105/ BCRC 12266), L. plantarum subsp. plantarum (ATCC 14917/BCRC 10069), Lactobacillus reuteri (ATCC 23272/BCRC 14625), Sporolactobacillus inulinus (ATCC 15538/BCRC 14647), and Streptococcus salivarius subsp. thermophilus (ATCC 19258/BCRC 13869), and Lactobacillus acidophilus (ATCC 53544/BCRC 17009) were purchased from the Bioresource Collection and Research Center (BCRC) in Taiwan. L. rhamnosus GG (LGG ${ }^{\circledR}$ Chr. Hansen, Denmark), L. reuteri (BioGaia ${ }^{\circledR}$, Sweden), and L. acidophilus (Infloran ${ }^{\circledR}$, Italy) were isolated from commercial products. Bacillus coagulans (BC1031) and Lactobacillus acidophilus (LA1063) were isolated from pure bacterial lyophilized powder individually purchased from Synbiotech (Taiwan). Lactobacillus rhamnosus GG (DSMZ 32550) and L. reuteri (BR101) were isolated from pure bacterial lyophilized powder that was purchased from Bio-Ray Biotech (Taiwan) individually. Anaerobiosis was achieved by incubating agar plates within an anaerobic jar (Mitsubishi Gas Chemical America, R685025, New York, NY, USA) with an anaerobic gas pack (Mitsubishi Gas Chemical America, R681001, New York, NY, USA) and incubating the liquid culture with mineral oil and 0.05\% L-cysteine (Sigma-Aldrich, 168149, St. Louis, MO, USA).

\subsection{In Vitro Bacterial Co-Culture}

To fulfill the growth criteria of the ten probiotic strains and two VRE strains, they were co-cultured in MRS broth under anaerobic static condition. VREfs and VREfm were inoculated in the MRS broth with a starting $\mathrm{OD}_{600}$ of 0.05 and incubated with agitation one day before the co-culture with probiotics in order to establish an Enterococcus predominant environment for the subsequently introduced probiotic strains similar to that of human gut of VRE carrier. The next day, probiotic strains, with a density of $1.67 \times 10^{6} \mathrm{CFU} / \mathrm{mL}$ each, were added to the bacterial cultures of VREfs and VREfm in MRS broth under anaerobic conditions. CFU concentrations of VREfs and VREfm were determined at fixed time points after introducing probiotic strains to evaluate the effect of the strains. The viability of the VRE population at a given time point was determined by counting the number of VRE CFUs after harvesting $100 \mu \mathrm{L}$ of the total bacterial culture and diluting it to a proper concentration to plate out on a selective medium (CHROMagar ${ }^{\mathrm{TM}}$ VRE, CHROMagar, VR952, Paris, France). The experiments were independently performed in duplicate.

\subsection{Bacterial DNA Isolation and Taxonomic Analysis}

In order to isolate total bacterial genomic DNA from the in vitro co-culture, $1.5 \mathrm{~mL}$ of the total bacterial mixture were harvested at indicated time points and centrifuged at $5000 \times g$ for $5 \mathrm{~min}$. The resultant bacterial pellets of two independent experiments were subjected to DNA isolation by using the bacterial genomic DNA purification kit (GeneMark, DP025, Taichung, Taiwan). The total bacterial genomic DNA samples that were obtained at individual time points were then dissected with 16S metagenomics sequencing (V3-V4 regions of 16S rRNA) to reveal the identity of individual bacterial strains and their proportion in the total population (Taipei Medical University Sequencing Core Facility). Next-generation sequencing (NGS) was performed while using the Illumina MiSeq System PE250 (San Diego, CA, USA). Raw data derived from PE250 sequencing were analyzed through the QIIME 1 pipeline [31]. Closed-reference picking and the Greengenes bacterial database [32] with 97\% sequence identity were used to cluster the operational taxonomic units (OTUs) in each sample. 


\subsection{Microbial Network Analysis}

The OTU tables from the QIIME analysis were exported in a tab-delimited format. Information from output tables was summarized according to taxonomy at the genus and species level. Forms were then analyzed while using the RMN algorithm [30] to infer microbial interactions that entailed competitive and cooperative relationships between VRE and probiotics. All of the parameters from the RMN pipeline followed original settings, except for the cut-off $\mathrm{D}$ value, which was set as 1 . In addition, some probiotics that had cooperative relationships with the VRE or competitive relationships with other probiotics were not considered.

\subsection{RNA Isolation and Quantitative Real-Time PCR}

Before the quantification of mRNA expression by using qRT-PCR, RNA was isolated using the total RNA purification kit (GeneMark, TR01, Taichung, Taiwan) from bacterial pellets after centrifugation of bacterial co-cultures, according to manufacturer's instructions. After extraction, the concentrations of RNA samples were determined using DeNovix DS-11+Spectrometer (DeNovix, Wilmington, DE, USA). After determining the quality and concentration of RNA samples by measuring the $\mathrm{OD}_{260 / 280}$, $1 \mu \mathrm{g}$ of RNA was treated with DNase I (AMPD1, Sigma-Aldrich, St. Louis, MO, USA) to remove any contaminated chromosomal DNA and then reverse-transcribed into cDNA using the iScript ${ }^{\mathrm{TM}} \mathrm{cDNA}$ Synthesis Kit (Bio-Rad, 1708891, Hercules, CA, USA). Quantitative real-time PCR was then performed by applying synthesized cDNA samples, corresponding primer pairs (Table S2), and SYBR ${ }^{\circledR}$ Green Master Mix (Bio-Rad, 1708886, Hercules, CA, USA) in triplicate to the CFX96 Real-Time System (Bio-Rad, Hercules, CA, USA). The comparative $\mathrm{Ct}\left({ }^{\Delta \Delta} \mathrm{Ct}\right)$ system was used to determine the relative mRNA expressions of selected VREfm virulence genes. The acquired mRNA expressions were normalized to the level of an internal control (16S rRNA of E. faecium) of each sample; subsequently, the fold changes against the corresponding control groups were calculated and expressed as $\log _{2}$ fold change.

\subsection{Caco-2 Cell Culture}

The Caco-2 cell line was purchased from the Bioresource Collection and Research Centre Taiwan (BCRC No. 67001, originally from [ATCC No. HTB-37] and routinely cultured in Dulbecco's modified Eagle's minimal essential medium (DMEM) (12800-017, Gibco, ThermoFisher Scientific, Waltham, MA, USA) with 10\% fetal bovine serum (10437-028, Gibco, ThermoFisher Scientific, Waltham, MA, USA) at $37^{\circ} \mathrm{C}$ and under $5 \% \mathrm{CO}_{2}$; media were changed every two to three days. For in vitro VREfm adherence assays, the Caco- 2 cells were seeded in 12-well cell culture plates (150628, Nunc, Waltham, MA, USA) at a density of $0.1 \times 10^{6}$ cells/well and maintained by replacing the media every two to three days to reach a complete confluency at $1 \times 10^{6}$ cells/well after 10 days.

\subsection{VREfm Adherence to Caco-2 Cells In Vitro}

The adherence of VREfm to Caco- 2 cells was inhibited using probiotics according to a previous procedure, with modifications [26]. For the exclusion assay, Caco-2 cells in each well of 12-well plates forming confluent monolayers were washed twice with Dulbecco's phosphate buffer saline (DPBS) (D5652, Sigma-Aldrich, St. Louis, MO, USA) and cultured with probiotic strains (approximately $3.33 \times 10^{8} \mathrm{CFU} / \mathrm{mL}$ ) for $1 \mathrm{~h}$. After incubation with probiotics, the Caco-2 cells were washed with DPBS twice and incubated with VREfm (approximately $3.33 \times 10^{6} \mathrm{CFU} / \mathrm{mL}$ ) for another $1 \mathrm{~h}$. Subsequently, the Caco-2 cells were washed twice with DPBS and then incubated with $0.05 \%$ Triton-X100 (T8787, Sigma-Aldrich, St. Louis, MO, USA) at $37^{\circ} \mathrm{C}$ for $20 \mathrm{~min}$. The adherent VREfm were then harvested with Caco-2 cell lysates and plated out on selective media after appropriate serial dilutions. For the displacement assay, the Caco-2 cells were initially cultured with VREfm for $1 \mathrm{~h}$ and then incubated with probiotics for another $1 \mathrm{~h}$. The rest of the procedures remained the same as in the exclusion assay. For the competition assay, Caco-2 cells were initially washed twice with DPBS and then co-incubated with probiotic strains (approximately $3.33 \times 10^{8} \mathrm{CFU} / \mathrm{mL}$ ) and VREfm (approximately 
$3.33 \times 10^{6} \mathrm{CFU} / \mathrm{mL}$ ) for $2 \mathrm{~h}$. After co-incubation, Caco-2 cells were washed twice to remove unattached bacterial cells and then subjected to the same cell lysis and plating out procedures. VREfm and probiotics of appropriate bacterial cell numbers were all resuspended in serum-free DMEM after determining their CFU concentrations before incubation with Caco-2 cells. The efficacies of probiotic treatment were compared with those of the control group without probiotics for the CFU number of VREfm remained attached to Caco-2 cells after assays. The experiments were independently performed in triplicate.

\subsection{VREfm and Probiotics Co-Cultured with Caco-2 Cells}

The Caco-2 cells were seeded in T75 cell culture flasks (156499, Nunc, ThermoFisher Scientific, Waltham, MA, USA) in advance at a cell density of $1.7 \times 10^{6}$ cells/flask and cultured for 10 days to reach confluency at $1.7 \times 10^{7}$ cells/flask. The CFU concentrations of VREfm and probiotic strain (B. coagulans_BC1031, L. rhamnosus GG_DSMZ 32250, and L. reuteri_BCRC BR101, and L. acidophilus_LA1063) inocula prepared one day in advance were determined. The inocula of VREfm and four probiotic strains were collected for an MOI of 350 to Caco-2 cells and washed twice with DPBS and then resuspended with serum-free DMEM. Caco-2 cells in the T75 flask were washed twice with DPBS and then co-incubated with VREfm and the probiotic combination for $2.5 \mathrm{~h}$ at $37^{\circ} \mathrm{C}$ under $5 \% \mathrm{CO}_{2}$. Bacterial cells of VREfm in supernatants were then harvested and washed twice with DPBS before RNA isolation. The same amount of VREfm in the absence of probiotic combination was incubated with Caco-2 cells in a T75 flask in parallel and collected as a control group. The same amount of VREfm that was incubated under the same culture condition without contacting Caco-2 cells was harvested as another control group.

\subsection{Statistical Analysis}

All of the data are presented as means and analyzed while using GraphPad Prism (version 5.0) (GraphPad Software Inc., La Jolla, CA, USA). Student's t-test was used to determine significant differences between the experimental groups; different $p$ values between paired data indicated the following levels of significance. $p<0.05,<0.01$, and $<0.001$ indicated statistically significant, highly significant, and extremely significant differences, respectively ${ }^{*} p<0.05,{ }^{*} p<0.01$, and $\left.{ }^{* * *} p<0.001\right)$.

\section{Results}

\subsection{Probiotic Mixture Significantly Reduced VRE Number in an In Vitro Co-Culture}

Ten strains of probiotic bacteria belonging to the genera of Bacillus, Bifidobacterium, Lactobacillus, Lactococcus, Sporolactobacillus, and Streptococcus were included in this study to determine a formula of probiotics to decolonize VRE in the human gut, as follows: Bacillus coagulans, Bifidobacterium bifidum, Bifidobacterium longum subsp. infantis, Lactococcus lactis subsp. lactis, Lactobacillus plantarum subsp. plantarum, Sporolactobacillus inulinus, Streptococcus salivarius subsp. thermophilus, Lactobacillus rhamnosus GG, Lactobacillus reuteri, and Lactobacillus acidophilus. The phenotypes of of VRE strains in vancomycin resistance were confirmed by disk diffusion assay and E-TEST following the guideline of CSLI (Table S3) in advance. van A was also detected in both strains with the gene-specific primer pair (Figure S1). The potential of inhibiting VRE was then investigated by co-culturing the mixture of ten probiotic strains with the inocula containing both VREfs and VREfm. The mixture of the ten probiotic strains expedited the decline of VREfs and VREfm populations, when compared with that observed in the group without probiotics, according to results shown in Figure 1a. The total bacterial pellets of 11 time points within three days of VRE-probiotic co-cultures were collected for genomic DNA isolation to identify the most critical probiotic strains and downsize the formula for outcompeting VRE. The fluctuations of bacterial populations during co-culture were detected through NGS, which revealed the V3-V4 region sequences of bacterial 16S rRNA and therefore 
the identities of all bacterial strains and their relative abundances. VRE constituted over $90 \%$ of the total population at the beginning of the co-culture, which decreased to approximately $60 \%$ between 15 and $48 \mathrm{~h}$ after co-culture and further declined to $30 \%$ after $64 \mathrm{~h}$. By contrast, L. rhamnosus, Lactobacillus_S, and Lactobacillus_other dominated at the end of co-culture (Figure 1b). The results of NGS were initially analyzed with the QIIME 1 pipeline and then clustered for OTUs in each sample. The constructed OTUs were then subjected to MNA based on a special algorithm, the RMN algorithm, which suggested the directions of bacterial competitive and cooperative relationships, helping to predict the most critical species during the co-culture and interactions between them. According to the MNA results, B. coagulans, L. reuteri, L. rhamnosus GG, and another Lactobacillus species were critical for suppressing the population of VRE, among which L. reuteri played the most important role (Figure 1c).
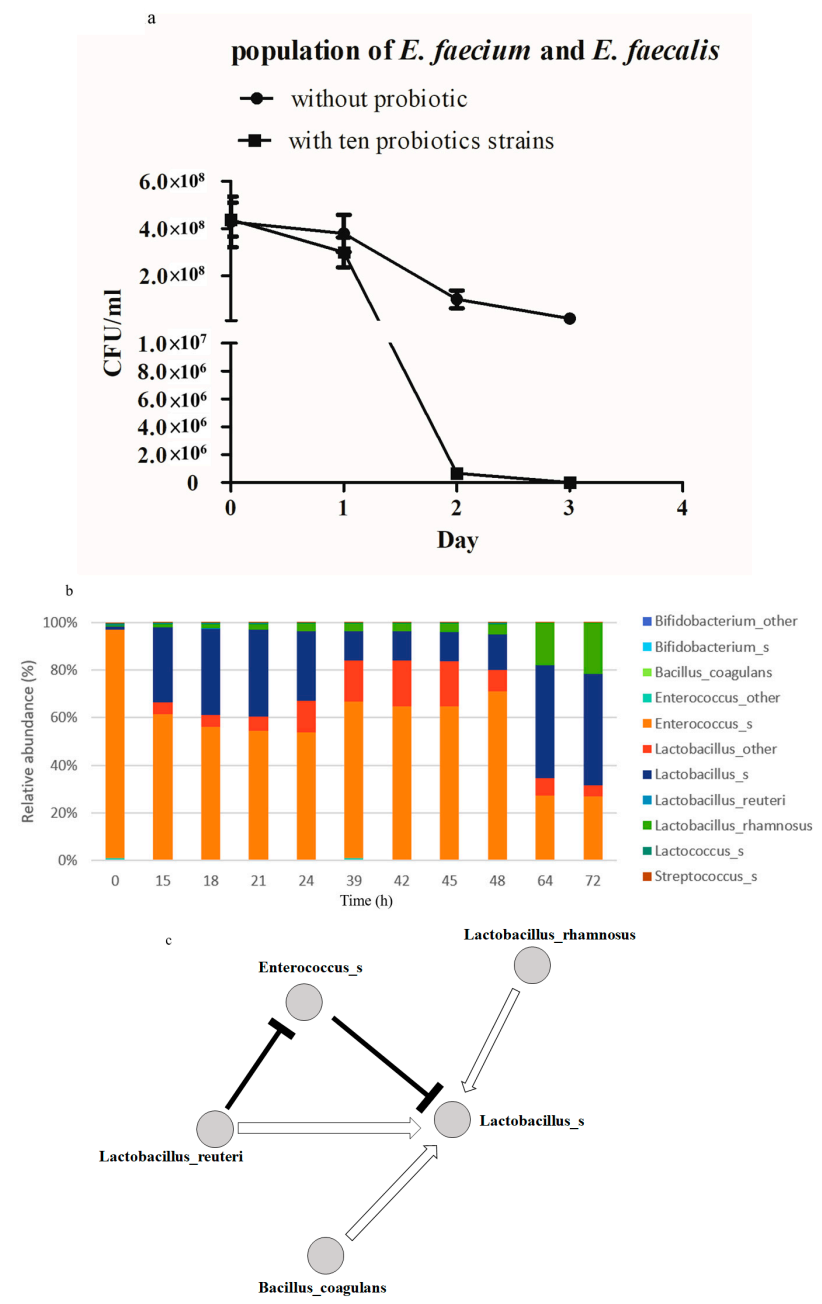

Figure 1. Probiotic mixture enhanced reduction of vancomycin-resistant enterococci (VRE) CFU in in vitro culture. Overnight-incubated VRE (E. faecalis and E. faecium) in the MRS broth were supplemented with or without a mixture of ten probiotic strains under anaerobic conditions for three days. (a) CFU concentrations of E. faecalis and E. faecium in co-culture mixture were determined daily and compared between the ten-probiotic co-culture group and control group without probiotic. (b) The compositions of the total bacterial population at 11 time points after co-culture revealed by 16S rRNA sequencing: each bacterial strain has been allotted a different-colored block in bar charts, with size that is proportional to its percentage in the total population at given time point. Note that, as the sequence of the V3-V4 region of $16 \mathrm{~S}$ rRNA may have been insufficient to distinguish bacterial species of the same genus, some of the bacterial species were numerated with only genus. In addition, one of the probiotic strains, namely S. inulinus, was not detected. (c) Microbial network analysis conducted based on the results of $16 \mathrm{~S}$ rRNA sequencing results to identify dominant probiotic strains 
during co-culture with VRE. Closed circles represent different bacterial species in the VRE-probiotic co-culture. Black-mallet-head lines indicate competitive relationships (mallet head pointed to the species being competed), whereas white arrows indicate cooperative relationships. Experiments of bacterial co-culture and its derived $16 \mathrm{~S}$ rRNA sequencing analysis were independently performed in duplicate.

The efficacy of these two strains against the VRE population in co-cultures was compared in combination with B. coagulans, L. rhamnosus GG, and L. reuteri to determine whether the unknown Lactobacillus species was L. plantarum or L. acidophilus. The combination of B. coagulans, L. rhamnosus GG, L. reuteri, and L. acidophilus was more effective than the combination of B. coagulans, L. rhamnosus GG, L. reuteri, and L. plantarum in reducing VRE concentrations, according to the results of co-culturing (Figure 2a). This suggests that L. acidophilus was a more promising component than L. plantarum in our probiotic formula.

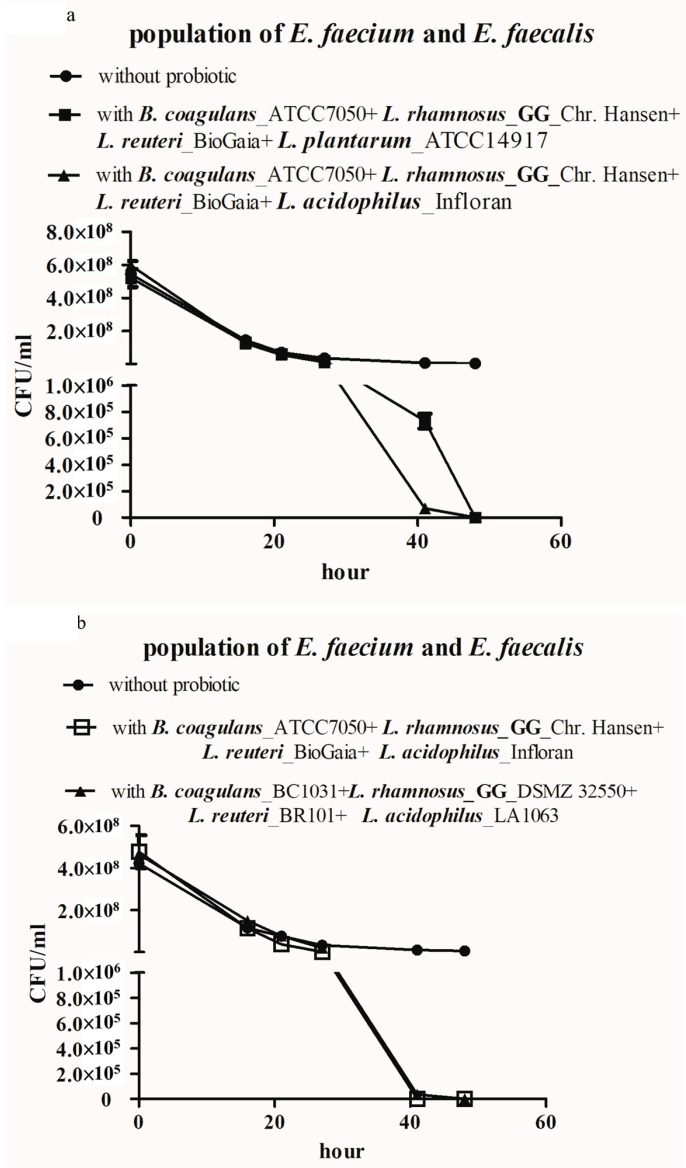

Figure 2. Microbial network analysis (MNA)-assisted identification of critical species for inhibiting VRE from the probiotic mixture. (a) The efficacies of L. plantarum_ATCC14917 and L. acidophilus_Infloran in repressing the co-cultured VRE population when in combination with B. coagulans_ATCC7050, L. rhamnosus GG_Chr. Hansen, and L. reuteri_BioGaia were compared. (b) Efficacy of suppressing co-cultured VRE population was compared between a combination of probiotic strains B. coagulans_ATCC7050, L. rhamnosus GG_Chr. Hansen, L. reuteri_BioGaia, and L. acidophilus_Infloran and the combination substitutes of the four probiotic strains, BC1031, DSMZ 32550, BR101, and LA1063.

In order to avoid the possibility of patent infringement, similar strains from alternative sources were adopted, as the three Lactobacillus species in our formula were isolated from commercial products (Table S1). ATCC originated-Lactobacillus rhamnosus GG (ATCC 53103/BCRC 16000), Lactobacillus reuteri (ATCC 23272/BCRC 14625), and Lactobacillus acidophilus (ATCC 53544/BCRC 17009) were purchased from 
BCRC. Samples of B. coagulans_BC1031 and L. acidophilus_LA1063 were purchased from SynBiotech (Taiwan), whereas samples of L.rhamnosus GG_DSMZ32,250 and L. reuteri_BCRC BR101 were purchased from Bio-Ray Biotech (Taiwan). The efficacies of the combination of the original four strains and the combination of their alternatives were then compared in VRE-probiotic co-cultures. B. coagulans_BC1031 in combination with Lactobacillus rhamnosus GG (ATCC 53103), Lactobacillus reuteri (ATCC 23272), Lactobacillus acidophilus (ATCC 53544) showed similar but relative weak activity in suppressing the co-cultured VRE when comparing to the original combination (Figure S2). The combination of B. coagulans_BC1031, L. rhamnosus_GG_DSMZ 32250, L. reuteri_BR101, and L. acidophilus_LA1063 showed effects that were comparable to those of the original combination with regard to reduction of VRE (Figure 2b). This result indicates that B. coagulans_BC1031 and L. rhamnosus GG_DSMZ 32250, L. reuteri_BCRC BR101, and L. acidophilus_LA1063 can be used as substitutes for original strains in further studies.

\subsection{MNA-Selected Four-Probiotic Mixture Reduced the Number of Co-Cultured VRE More Significantly than Did Individual Strains}

The efficacies of the mixture of the four probiotic strains in an equal ratio and individual strains were compared to further confirm the necessity of combining the four probiotic strains. In the VRE-probiotic co-culture model, a combination of B. coagulans_BC1031, L. rhamnosus GG_DSMZ 32250, L. reuteri_BCRC BR101, and L. acidophilus_LA1063 (Figure 3a, squares) surpassed B. coagulans_BC1031 (Figure 3a, diamonds) or L. rhamnosus GG_DSMZ 32,250 (Figure 3a, inverted triangles) alone in reducing VREfm and VREfs populations and showed a significant difference at the time point of $41 \mathrm{~h}$. Supplementing L. rhamnosus GG_DSMZ 32,250 alone did not significantly reduce the number of VRE until at the time point of $48 \mathrm{~h}$ (Figure 3a, inverted triangles), whereas the VRE population in the group that was supplemented with B. coagulans_BC1031 alone (Figure 3a, diamonds) did not show any difference from the VRE only group (Figure 3a, circles). The efficacies of L. reuteri_BCRC BR101 and L. acidophilus_LA1063 alone were also evaluated. L. reuteri_BCRC BR101 showed a relatively weaker efficacy than that of the combination of the four strains at 41 and $48 \mathrm{~h}$ (Figure 3b, squares vs. triangles), whereas L. acidophilus_LA1063 slightly enhanced the reduction of co-cultured VRE, but did not significantly diminish the majority of VRE (Figure 3b, diamonds).

Caco-2 cells, a well-established human epithelial colorectal adenocarcinoma cell line, have been widely utilized as an in vitro model in studying adherence of pathogenic or probiotic bacterial species to gut epithelial cells [26,33]. The efficacy of reducing VRE adherence to Caco-2 cells was compared between the four-strain probiotic combination and individual strains because L. reuteri reduces the adherence of pathogenic bacteria to Caco-2 cells [26]. Three different approaches were adopted to evaluate the effectiveness of probiotics in reducing the adherence of VRE. In the exclusion assay, probiotics were incubated with Caco-2 cells in advance and their effectiveness in blocking the attachment of VRE that was introduced later was evaluated; in the competition assay, probiotics were simultaneously supplemented with VRE and their efficacy of competing with VRE in adherence to Caco-2 cells was evaluated; and in the displacement assay, probiotics were introduced to Caco-2 cells that were pre-incubated with VRE and their effectiveness in displacing the attached VRE was evaluated [26]. All of the probiotic-introducing treatment would be compared with its counterpart control that without probiotic. We focused on VREfm, which was reported to show more severe antibiotic resistance, to simplify our study [34]. Supplementing probiotics as individual strains or in combination did not significantly prevent the attachment of the later introduced VREfm to Caco-2 cells, according to the results shown in Figure 3c. By contrast, when probiotics, except for L. acidophilus_LA1063, were supplemented along with VREfm, the attached VREfm of each group was reduced to $30-50 \%$ (Figure $3 \mathrm{~d}$ ). When probiotics were administered to Caco-2 cells pre-incubated with VREfm, the remaining attached VREfm reduced to $65-77 \%$ of that observed in the control group without probiotics, except for the one that was supplemented with L. acidophilus_LA1063 (Figure 3e). Although the combination of the four probiotic strains did not show significantly better efficacy than 
that of individual strains of B. coagulans_BC1031, L. rhamnosus GG_DSMZ 32250, or L. reuteri_BCRC BR101 in reducing the adherence of E. faecium to Caco-2 cells, it was more promising in reducing the VRE concentrations in co-cultures and, therefore, more suitable for subsequent studies.
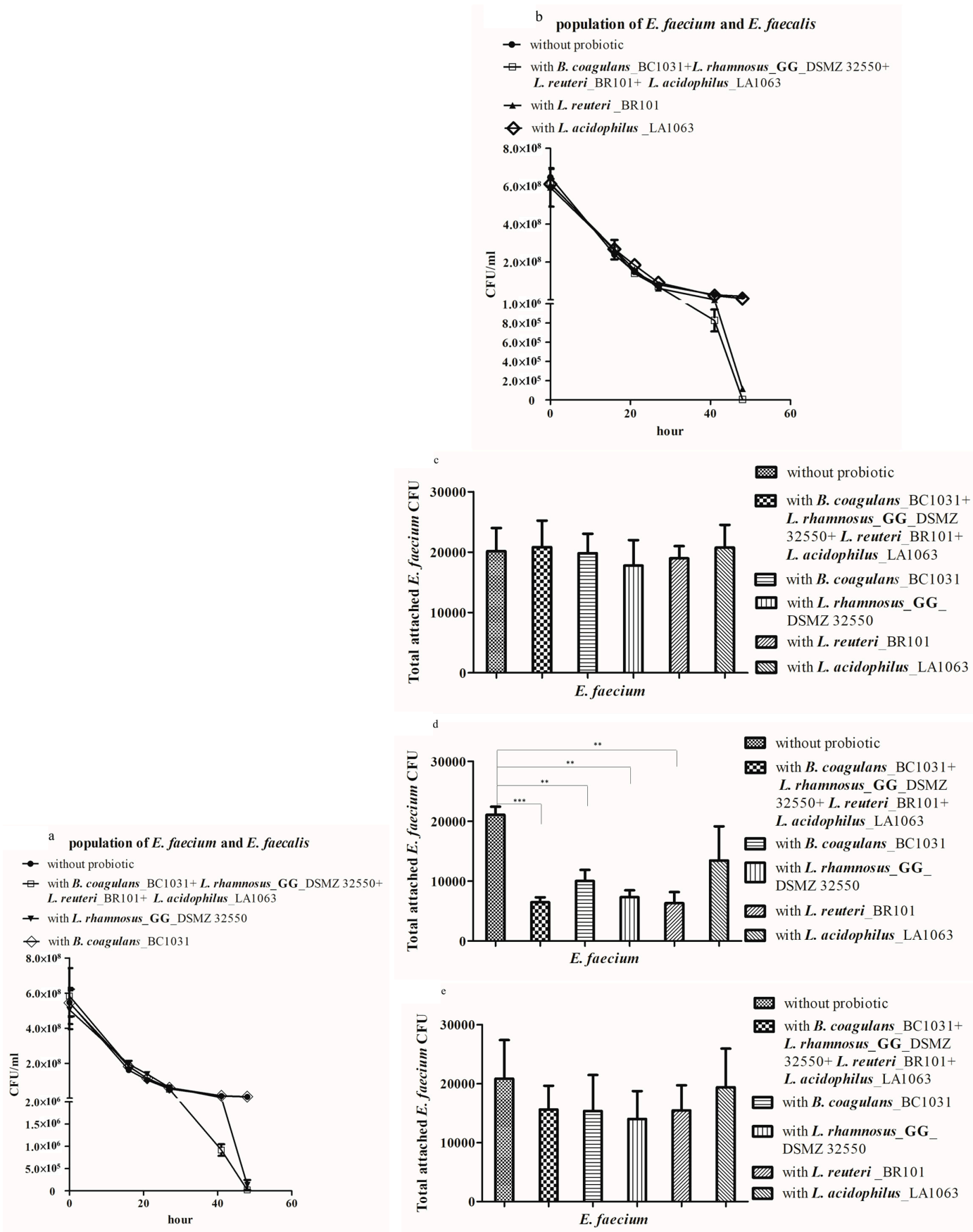

Figure 3. The combination of four probiotic strains showed better efficacy than that of individual strains against co-cultured VRE. Individual strains of (a) B. coagulans_ BC1031 or L. rhamnosus GG_ DSMZ 32550, (b) L. reuteri_ BR101 or L. acidophilus_ LA1063 were compared with the combination of the four strains for the efficacy against the co-cultured VREfs and VREfm population. (c) Efficacies of pre-incubated individual probiotics strains and that of the combination of four probiotic strains were compared with exclusion assay. (d) Competition assay was performed to compare the ability of individual probiotics strains and combination of the four probiotic strains in competing with E. faecium for binding to Caco-2 cells. (e) Comparison of the efficacies of individual strains and the combination of the four strains in displacing E. faecium adherent to Caco-2 cells. The probiotic combination used here 
consisted of the four strains in equal ratio. The experiments of bacterial co-cultures were independently performed in duplicate, whereas the experiments of VRE adherence to Caco-2 cells were independently performed in triplicate. $<0.01$, and $<0.001$ indicated statistically significant, highly significant, and extremely significant differences, respectively and depicted as ${ }^{* *} p<0.01$, and ${ }^{* * *} p<0.001$ in figures.

\subsection{The Four-Probiotic Mixture Significantly Downregulated the Expression of Nine Virulence Genes of VREfm}

The probiotic combination showed significant efficacy in competing with VREfm for binding to Caco-2 cells. This implies that virulence genes that are involved in bacterial adherence to host cells might be affected during interactions between VREfm and probiotics. The expression of VREfm virulence genes in the presence of host cells and interaction with probiotics were evaluated by co-culturing VREfm and the four-strain probiotic mixture in equal ratios with Caco-2 cells for $2.5 \mathrm{~h}$ in order to validate this hypothesis. The bacterial cells of VREfm were then harvested for quantifying the expressions of virulence genes associated with adherence to host cells. VREfm co-cultured with both Caco-2 cells and probiotics was compared with VREfm co-cultured with only Caco-2 cells and with VREfm not contacting Caco-2 cells in the expression levels of the selected nine virulence genes. Nine adherence-related genes were included in this study. $a c m$ encodes the adhesin to facilitate the binding of type I and IV collagen [35]. $e b p A, e b p B$, and $e b p C$ are the genes encoding for subunits of E. faecium pilus that are required for fibrinogen binding [36,37]. EfaA shares $42-63 \%$ similarity of amino acid sequences with a family of streptococcal virulence and adhesion proteins [38]. SagA is capable of adhering to a wide range of extracellular proteins [39]. Esp, which is an important antigenic marker of endocarditis, is required for biofilm formation [40]. sgrA encodes an adhesion protein with the LPXTG motif and it is potentially required for biofilm formation [41]. scm encodes a collagen-binding protein [37]. The results of our study demonstrated that $a c m$, sagA, and esp exhibited similar mRNA expressions after contacting Caco-2 cells (Figure 4a,f,g). The mRNA expressions of $e b p A, e b p B$, and $e b p C$ slightly increased to approximately two-fold (Figure $4 \mathrm{~b}-\mathrm{d}$ ). Meanwhile, the mRNA expressions of efa $A$, $s g r A$, and $s c m$ decreased two- to four-fold after contacting Caco-2 cells (Figure 4e,h,i). In addition, all of the nine virulence genes of VREfm were significantly downregulated after co-culturing with Caco-2 cells in the presence of the four-strain probiotic mixture. These results suggest that adherence-associated genes that are responsible for different host environments may act differently under a given condition, and their mRNA expressions were suppressed when VREfm interacting with the four-strain probiotic mixture, thus reducing the adherence of VREfm to Caco-2 cells. 


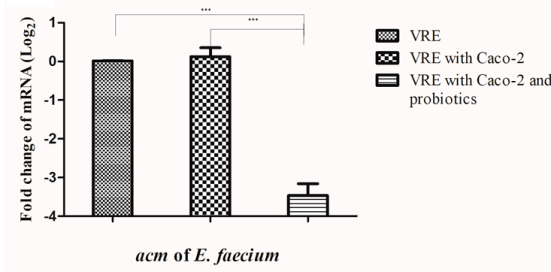

(a)

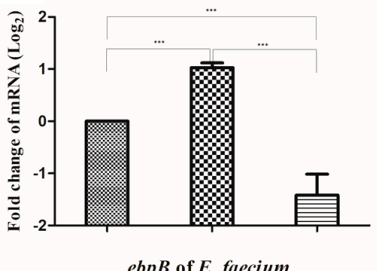

(c)

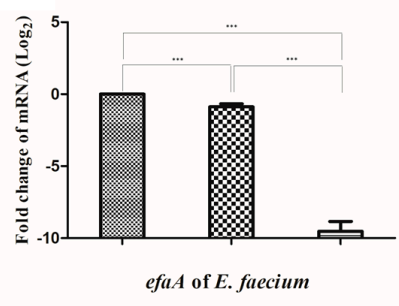

(e)

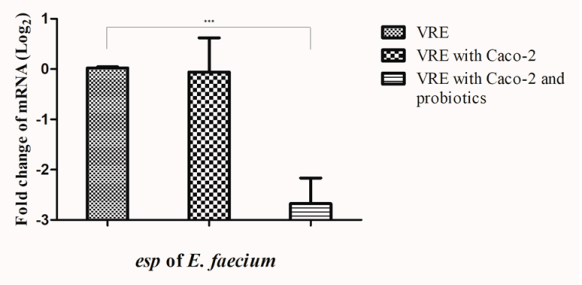

(g)

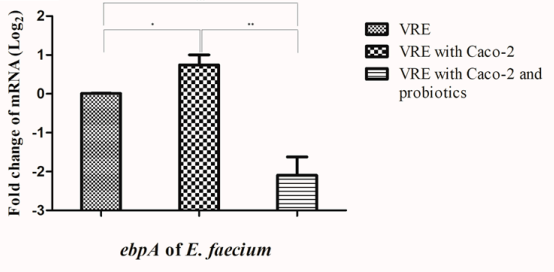

(b)

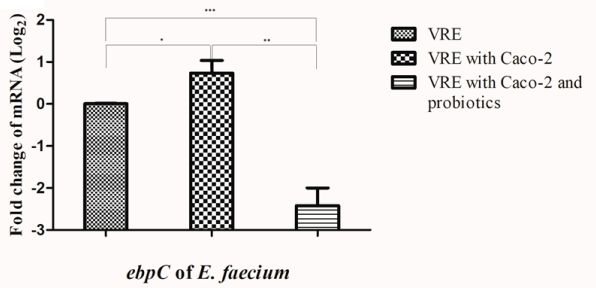

(d)

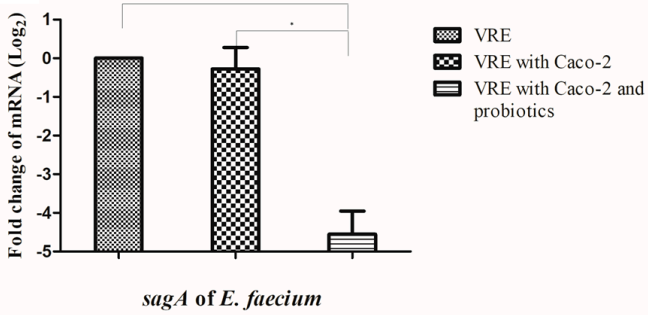

(f)

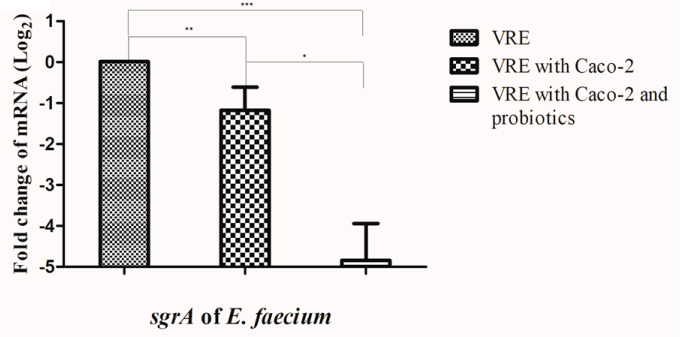

(h)

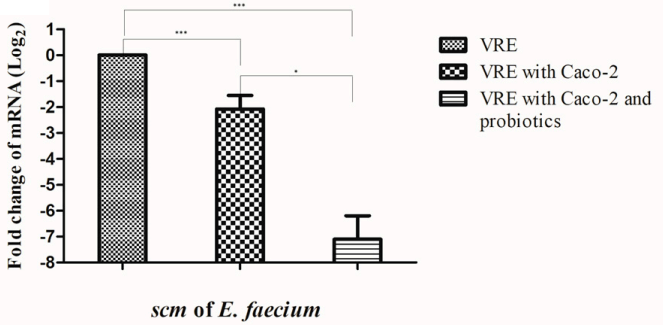

(i)

Figure 4. Probiotic combination reduced the expression of VRE E. faecium virulence genes when co-incubated with Caco-2 cells. The mRNA expression levels of E. faecium virulence genes: (a) $a \mathrm{~cm}$, (b) $e b p A,(\mathbf{c}) e b p B,(\mathbf{d}) e b p C,(\mathbf{e}) e f a A,(\mathbf{f}) \operatorname{sag} A,(\mathbf{g}) e s p$, (h) $\operatorname{sgr} A$, and (i) $s c m$ were evaluated after incubation in serum-free Dulbecco's modified Eagle's minimal essential medium (DMEM) alone (VRE), with Caco-2 cells (VRE and Caco-2), and with Caco-2 cells and probiotics (VRE with Caco-2 and probiotics) at $37^{\circ} \mathrm{C}$ under $5 \% \mathrm{CO}_{2}$ for $2.5 \mathrm{~h}$. The experiments were independently performed in triplicate. $p<0.05,<0.01$, and $<0.001$ indicated statistically significant, highly significant, and extremely significant differences, respectively and depicted as ${ }^{*} p<0.05,{ }^{* *} p<0.01$, and ${ }^{* * *} p<0.001$ in figures. 


\section{Discussion}

Discovery of alternative antibacterial drugs and therapies is warranted owing to the emergence of multiple-drug-resistant bacteria and the shortage of new antibiotics. The cooperation and competition between microorganisms can be exploited to develop main or supportive therapies $[42,43]$. We used MNA driven by the RMN algorithm and found that the combination of B. coagulans, L. rhamnosus GG, L. reuteri, and L. acidophilus significantly repressed the co-cultured VRE population, reduced the attachment of VREfm to Caco-2 cells, and downregulated the mRNA expression of VREfm virulence genes. To the best of our knowledge, this is the first study in which multiple probiotic species were used in order to prevent the colonization of VRE in the human intestinal epithelial cell line, suggesting the feasibility of decolonizing VRE in the human gut.

A study reported that a combination of probiotic species decolonized extended spectrum beta-lactamase-producing Enterobacteriaceae in a randomized clinical trial, [44] and led to better outcomes than those that were yielded by single species in clinical trials of modulating gut microflora and eradicating H. pylori infection [29]. This might be attributed to the mutualistic or commensalistic relationships between probiotic species [45]. All probiotic species in the four-probiotic mixture have been reported to possess bioactivities that help fight against VRE; administering B. coagulans reduced the colonization of VRE in a mouse model [46]; supplementing L. rhamnosus GG significantly decolonized VRE in participants in two independent clinical trials [27,47]; L. reuteri was effective in reducing the attachment of VRE to Caco-2 cells [26]; and, L. acidophilus enhanced the host immune response of C. elegans against E. faecalis infection [48]. In our study, these four probiotic strains showed relatively weak or no efficacies in reducing the co-cultured VRE population individually, when comparing with the efficacy of their combination. This might be attributed to the synergistic effect of cooperative relationships among these four strains (Figure 3a,b), as suggested by MNA based on the RMN algorithm in this study. The RMN algorithm is a rule-based microbial network algorithm that not only suggests cooperative and competitive relationships between microorganisms, but also infers the directions of such relationships; therefore, the algorithm helps to suggest the most pivotal species by reconstructing regulatory networks in a complex microbial community [30]. In addition, RMN proved beneficial in dissecting the interactions among neutrophilic iron-oxidizing Zetaproteobacteria and constructing microbial networks in the hydrothermal vent ecosystems [49]. MNA was useful in identifying the most critical probiotic strains among the ten selected probiotic strains. Given that the NGS data of the V3-V4 region of total bacterial 16S rRNA failed to reveal the specific species of all Lactobacillus strains, the MNA still suggested that $L$. reuteri and L. rhamnosus GG play important roles; this helped to reduce the time that is required for confirmation. After switching to alternative sources of the four probiotic strains (Figure $2 \mathrm{~b}$ and Figure S2), the alternative four strains still generated similar results, which suggested that the effect of these four strains on VRE is not specific to the strains from specific sources and consolidates the choice of these four strains.

Antibiotic treatment to VRE carriage is hampered by the intrinsic resistance of enterococci, which is contributed by a hardened cell wall and the accumulation of antibiotic resistance genes, and might facilitate VRE overgrowth and resistance gene transmission [50]. Thus, oral administration of probiotics is an alternative that was evaluated in several studies. In trials which were conducted by Manley et al. and Szachta et al., administration of L. rhamnosus GG significantly reduced VRE colonization in trial participants [27,47]. However, a similar approach did not yield positive results in the trial that was conducted by Doron et al. [28]. Lcr35, an L. rhamnosus probiotic strain phylogenically close to LGG [51], did not have a significant effect on VRE carriage [52]. These controversial results may be attributed to different operation protocols (e.g., probiotic strains, dosage, and duration) and cohort size, as well as to the gut microbiome diversity between the cohorts of different trials, which could profoundly influence the colonization of probiotic species [53]. The strategy of administering multiple probiotic species, including L. rhamnosus and L. acidophilus, was adopted in another trial; however, it failed to prevent the colonization of ampicillin-resistant E. faecium [54], which suggested that MNA-assisted, in vitro study-supported selection of a mixture of multiple probiotics can be more promising in clinical trials. 
Fecal microbiota transplantation (FMT) to decolonize VRE can also be regarded as a multiple-species treatment that reduced the colonization of VRE in a mouse model and a clinical trial $[55,56]$. However, FMT treatment is more invasive and it carries a risk of multiple-drug-resistant pathogenic infections. Therefore, the administration of probiotics would be a safer option than FMT for VRE-carrying patients with other underlying diseases or compromised immune system.

Adhesion molecules are important for the colonization and dissemination of enterococci in the host environment [57]. In our study, decreased colonization by VRE in Caco-2 cells was observed under treatment with the four-probiotic mixture, accompanied by the downregulation of adherence-associated genes. Nine adherence-associated genes that were included in this study exhibited differential responses after incubation with Caco-2 cells and they were significantly downregulated when the four-strain probiotic mixture was incubated with VREfm (Figure 4a-i). A similar trend in the downregulation of these nine genes might be a coordinated global regulation. In E. faecalis, the homologous genes of $e b p A, e b p B, e b p C$, and $e f a A$ were reported to be regulated by FsrABC [58,59], a quorum-sensing system that regulates various virulence genes [60]. FsrABC shares sequence homology with the Staphylococcus aureus AgrABC system [60], which can be inhibited by fengycins that are produced by Bacillus species [23]. Therefore, the likelihood of FsrABC, the AgrABC-like quorum-sensing system in E. faecalis and E. faecium, being regulated by oligopeptides or other secretory molecules produced by the four-strain probiotic mixture and downregulating the expressions of various adhesion genes is worth investigating. Whether all of the nine adherence-associated genes are under regulation of FsrABC warrants further investigation. In addition to the downregulation of VRE adherence-associated genes, other underlying mechanisms may also contribute to the reduced attachment of VRE to Caco-2 cells. LGG possesses a pilus gene cluster that shares sequence similarity with the pilus gene cluster of VRE; therefore, preventing VRE from attaching to mucus by direct competition [25]. Bacteriocin produced by probiotic species contributes to the reduction of VRE colonization [61]. In addition, Lactobacillus species induced the expression of antimicrobial peptide human LL-37 and reduced VRE persistence in mice [62]. Whether the four-probiotic mixture adopts similar mechanisms remains unclear.

\section{Conclusions}

In conclusion, we introduced an MNA-selected four-probiotic mixture that was effective in inhibiting co-cultured VRE and reducing the attachment of VRE to Caco-2 cells in vitro. The mixture is being used in the first clinical trial that aims to decolonize VRE in the human gut by using multiple probiotic species in order to validate the efficacy of this four-probiotic mixture in the human gut (ClinicalTrial.gov ID: NCT03822819). The mechanism of action of this four-probiotic mixture should be investigated in additional prospective studies, and these studies can provide clues for potential optimization of this mixture in the future.

Supplementary Materials: The following are available online at http://www.mdpi.com/2076-2607/8/6/816/s1, Figure S1: vanA detection in VREfs and VREfm of this study, Figure S2: Efficacy comparison between the four-strain probiotic combination of different sources, Table S1: Strains used in this study and their culture conditions, Table S2: Primers used in this study, Table S3: Disk diffusion assay result of VRE strains in this study.

Author Contributions: Conceptualization, S.-B.F. and Y.-J.L.; methodology, W.-S.S.; K.N.T.; software, K.-N.T. and Y.-H.H.; formal analysis, W.-S.S.; resources, S.-B.F., K.-N.T., and Y.-H.H.; data curation, W.-S.S.; writing-Original draft preparation, W.-S.S.; writing-Review and editing, S.-B.F., K.-N.T., and Y.-H.H.; visualization, Y.-J.L.; supervision, Y.-J.L.; project administration, S.-B.F.; funding acquisition, S.-B.F., and K.-N.T. All authors have read and agreed to the published version of the manuscript.

Funding: This study was funded by the Delta-TMU SHH Industry-Academy Cooperation Project (A-106-053, A-107-061).

Acknowledgments: We thank Wen-Shyane Hsieh for providing the clinical isolate of Enterococcus faecalis and the Sequencing Core Facility Center, Taipei Medical University, for their assistance in performing $16 \mathrm{~S}$ rRNA sequencing.

Conflicts of Interest: The founder of this study had no role in the study design, data collection and interpretation, or the decision to submit the manuscript for publication. We have applied for patents for this research in Taiwan (Application No.108131776), China (Application No. 2019108310244), and USA (Application No. 16/818661) that are currently under review. 


\section{References}

1. Schloissnig, S.; Arumugam, M.; Sunagawa, S.; Mitreva, M.; Tap, J.; Zhu, A.; Waller, A.; Mende, D.R.; Kultima, J.R.; Martin, J.; et al. Genomic variation landscape of the human gut microbiome. Nature 2013, 493, 45-50. [CrossRef] [PubMed]

2. Van Tyne, D.; Gilmore, M.S. Friend turned foe: Evolution of enterococcal virulence and antibiotic resistance. Annu. Rev. Microbiol. 2014, 68, 337-356. [CrossRef] [PubMed]

3. De Almeida, L.M.; de Araujo, M.R.; Iwasaki, M.F.; Sacramento, A.G.; Rocha, D.; da Silva, L.P.; Pavez, M.; de Brito, A.C.; Ito, L.C.; Gales, A.C.; et al. Linezolid resistance in vancomycin-resistant Enterococcus faecalis and Enterococcus faecium isolates in a Brazilian hospital. Antimicrob. Agents Chemother. 2014, 58, 2993-2994. [CrossRef] [PubMed]

4. Palmer, K.L.; Kos, V.N.; Gilmore, M.S. Horizontal gene transfer and the genomics of enterococcal antibiotic resistance. Curr. Opin. Microbiol. 2010, 13, 632-639. [CrossRef] [PubMed]

5. Zhong, Z.; Zhang, W.; Song, Y.; Liu, W.; Xu, H.; Xi, X.; Menghe, B.; Zhang, H.; Sun, Z. Comparative genomic analysis of the genus Enterococcus. Microbiol. Res. 2017, 196, 95-105. [CrossRef]

6. O'Driscoll, T.; Crank, C.W. Vancomycin-resistant enterococcal infections: Epidemiology, clinical manifestations, and optimal management. Infect. Drug Resist. 2015, 8, 217-230. [CrossRef]

7. Courvalin, P. Transfer of antibiotic resistance genes between gram-positive and gram-negative bacteria. Antimicrob. Agents Chemother. 1994, 38, 1447-1451. [CrossRef]

8. Puchter, L.; Chaberny, I.F.; Schwab, F.; Vonberg, R.P.; Bange, F.C.; Ebadi, E. Economic burden of nosocomial infections caused by vancomycin-resistant enterococci. Antimicrob. Resist. Infect. Control. 2018, 7, 1. [CrossRef]

9. Kos, V.N.; Desjardins, C.A.; Griggs, A.; Cerqueira, G.; Van Tonder, A.; Holden, M.T.; Godfrey, P.; Palmer, K.L.; Bodi, K.; Mongodin, E.F.; et al. Comparative genomics of vancomycin-resistant Staphylococcus aureus strains and their positions within the clade most commonly associated with Methicillin-resistant S. aureus hospital-acquired infection in the United States. MBio 2012, 3. [CrossRef]

10. Kurenbach, B.; Bohn, C.; Prabhu, J.; Abudukerim, M.; Szewzyk, U.; Grohmann, E. Intergeneric transfer of the Enterococcus faecalis plasmid pIP501 to Escherichia coli and Streptomyces lividans and sequence analysis of its tra region. Plasmid 2003, 50, 86-93. [CrossRef]

11. Uttley, A.H.; George, R.C.; Naidoo, J.; Woodford, N.; Johnson, A.P.; Collins, C.H.; Morrison, D.; Gilfillan, A.J.; Fitch, L.E.; Heptonstall, J. High-level vancomycin-resistant enterococci causing hospital infections. Epidemiol. Infect. 1989, 103, 173-181. [CrossRef] [PubMed]

12. Gordon, S.; Swenson, J.M.; Hill, B.C.; Pigott, N.E.; Facklam, R.R.; Cooksey, R.C.; Thornsberry, C.; Jarvis, W.R.; Tenover, F.C. Antimicrobial susceptibility patterns of common and unusual species of enterococci causing infections in the United States. Enterococcal Study Group. J. Clin. Microbiol. 1992, 30, 2373-2378. [CrossRef] [PubMed]

13. Gold, H.S. Vancomycin-resistant enterococci: mechanisms and clinical observations. Clin. Infect. Dis. 2001, 33, 210-219. [CrossRef] [PubMed]

14. Billington, E.O.; Phang, S.H.; Gregson, D.B.; Pitout, J.D.; Ross, T.; Church, D.L.; Laupland, K.B.; Parkins, M.D. Incidence, risk factors, and outcomes for Enterococcus spp. blood stream infections: A population-based study. Int. J. Infect. Dis. 2014, 26, 76-82. [CrossRef] [PubMed]

15. Tacconelli, E.M.N. Global Priority List of Antibiotic-Resistant Bacteria to Guide Research, Discovery, and Development of New Antibiotics. Available online: https:/www.who.int/medicines/publications/globalpriority-list-antibiotic-resistant-bacteria/en/ (accessed on 29 May 2020).

16. Bhatt, P.; Sahni, A.K.; Praharaj, A.K.; Grover, N.; Kumar, M.; Chaudhari, C.N.; Khajuria, A. Detection of glycopeptide resistance genes in enterococci by multiplex PCR. Med. J. Armed Forces India 2015, 71, $43-47$. [CrossRef] [PubMed]

17. Murray, B.E. The life and times of the Enterococcus. Clin. Microbiol. Rev. 1990, 3, 46-65. [CrossRef] [PubMed]

18. Le Barz, M.; Anhe, F.F.; Varin, T.V.; Desjardins, Y.; Levy, E.; Roy, D.; Urdaci, M.C.; Marette, A. Probiotics as complementary treatment for metabolic disorders. Diabetes Metab. J. 2015, 39, 291-303. [CrossRef]

19. Macpherson, A.J.; Harris, N.L. Interactions between commensal intestinal bacteria and the immune system. Nat. Rev. Immunol. 2004, 4, 478-485. [CrossRef] 
20. Gourbeyre, P.; Denery, S.; Bodinier, M. Probiotics, prebiotics, and synbiotics: Impact on the gut immune system and allergic reactions. J. Leukoc. Biol. 2011, 89, 685-695. [CrossRef]

21. Kamada, N.; Chen, G.Y.; Inohara, N.; Nunez, G. Control of pathogens and pathobionts by the gut microbiota. Nat. Immunol. 2013, 14, 685-690. [CrossRef]

22. Fukuda, S.; Toh, H.; Hase, K.; Oshima, K.; Nakanishi, Y.; Yoshimura, K.; Tobe, T.; Clarke, J.M.; Topping, D.L.; Suzuki, T.; et al. Bifidobacteria can protect from enteropathogenic infection through production of acetate. Nature 2011, 469, 543-547. [CrossRef] [PubMed]

23. Piewngam, P.; Zheng, Y.; Nguyen, T.H.; Dickey, S.W.; Joo, H.S.; Villaruz, A.E.; Glose, K.A.; Fisher, E.L.; Hunt, R.L.; Li, B.; et al. Pathogen elimination by probiotic Bacillus via signalling interference. Nature 2018, 562, 532-537. [CrossRef] [PubMed]

24. Dembele, T.; Obdrzalek, V.; Votava, M. Inhibition of bacterial pathogens by lactobacilli. Zentralbl. Bakteriol. 1998, 288, 395-401. [CrossRef]

25. Tytgat, H.L.; Douillard, F.P.; Reunanen, J.; Rasinkangas, P.; Hendrickx, A.P.; Laine, P.K.; Paulin, L.; Satokari, R.; de Vos, W.M. Lactobacillus rhamnosus GG Outcompetes Enterococcus faecium via Mucus-Binding Pili: Evidence for a novel and heterospecific probiotic mechanism. Appl. Environ. Microbiol. 2016, 82, 5756-5762. [CrossRef]

26. Singh, T.P.; Kaur, G.; Kapila, S.; Malik, R.K. Antagonistic activity of Lactobacillus reuteri strains on the adhesion characteristics of selected pathogens. Front. Microbiol. 2017, 8, 486. [CrossRef]

27. Szachta, P.; Ignys, I.; Cichy, W. An evaluation of the ability of the probiotic strain Lactobacillus rhamnosus GG to eliminate the gastrointestinal carrier state of vancomycin-resistant enterococci in colonized children. J. Clin. Gastroenterol. 2011, 45, 872-877. [CrossRef] [PubMed]

28. Doron, S.; Hibberd, P.L.; Goldin, B.; Thorpe, C.; McDermott, L.; Snydman, D.R. Effect of Lactobacillus rhamnosus GG administration on vancomycin-resistant enterococcus colonization in adults with comorbidities. Antimicrob. Agents Chemother. 2015, 59, 4593-4599. [CrossRef]

29. Chapman, C.M.; Gibson, G.R.; Rowland, I. Health benefits of probiotics: Are mixtures more effective than single strains? Eur. J. Nutr. 2011, 50, 1-17. [CrossRef]

30. Tsai, K.N.; Lin, S.H.; Liu, W.C.; Wang, D. Inferring microbial interaction network from microbiome data using RMN algorithm. BMC Syst. Biol. 2015, 9, 54. [CrossRef]

31. Caporaso, J.G.; Kuczynski, J.; Stombaugh, J.; Bittinger, K.; Bushman, F.D.; Costello, E.K.; Fierer, N.; Pena, A.G.; Goodrich, J.K.; Gordon, J.I.; et al. QIIME allows analysis of high-throughput community sequencing data. Nat. Methods 2010, 7, 335-336. [CrossRef]

32. DeSantis, T.Z.; Hugenholtz, P.; Larsen, N.; Rojas, M.; Brodie, E.L.; Keller, K.; Huber, T.; Dalevi, D.; Hu, P.; Andersen, G.L. Greengenes, a chimera-checked $16 \mathrm{~S}$ rRNA gene database and workbench compatible with ARB. Appl. Environ. Microbiol. 2006, 72, 5069-5072. [CrossRef] [PubMed]

33. Tuomola, E.M.; Salminen, S.J. Adhesion of some probiotic and dairy Lactobacillus strains to Caco-2 cell cultures. Int. J. Food Microbiol. 1998, 41, 45-51. [CrossRef]

34. Weiner, L.M.; Webb, A.K.; Limbago, B.; Dudeck, M.A.; Patel, J.; Kallen, A.J.; Edwards, J.R.; Sievert, D.M. Antimicrobial-resistant pathogens associated with healthcare-associated infections: Summary of data reported to the national healthcare safety network at the centers for disease control and prevention, 2011-2014. Infect. Control. Hosp. Epidemiol. 2016, 37, 1288-1301. [CrossRef] [PubMed]

35. Nallapareddy, S.R.; Weinstock, G.M.; Murray, B.E. Clinical isolates of Enterococcus faecium exhibit strain-specific collagen binding mediated by Acm, a new member of the MSCRAMM family. Mol. Microbiol. 2003, 47, 1733-1747. [CrossRef]

36. Flores-Mireles, A.L.; Walker, J.N.; Potretzke, A.; Schreiber, H.L.; Pinkner, J.S.; Bauman, T.M.; Park, A.M.; Desai, A.; Hultgren, S.J.; Caparon, M.G. Antibody-based therapy for enterococcal catheter-associated urinary tract infections. MBio 2016, 7. [CrossRef]

37. Sillanpaa, J.; Nallapareddy, S.R.; Prakash, V.P.; Qin, X.; Hook, M.; Weinstock, G.M.; Murray, B.E. Identification and phenotypic characterization of a second collagen adhesin, $\mathrm{Scm}$, and genome-based identification and analysis of 13 other predicted MSCRAMMs, including four distinct pilus loci, in Enterococcus faecium. Microbiology 2008, 154, 3199-3211. [CrossRef]

38. Singh, K.V.; Coque, T.M.; Weinstock, G.M.; Murray, B.E. In vivo testing of an Enterococcus faecalis efaA mutant and use of efaA homologs for species identification. FEMS Immunol. Med. Microbiol. 1998, 21, 323-331. [CrossRef] 
39. Teng, F.; Kawalec, M.; Weinstock, G.M.; Hryniewicz, W.; Murray, B.E. An Enterococcus faecium secreted antigen, SagA, exhibits broad-spectrum binding to extracellular matrix proteins and appears essential for E. faecium growth. Infect. Immun. 2003, 71, 5033-5041. [CrossRef]

40. Heikens, E.; Singh, K.V.; Jacques-Palaz, K.D.; van Luit-Asbroek, M.; Oostdijk, E.A.; Bonten, M.J.; Murray, B.E.; Willems, R.J. Contribution of the enterococcal surface protein Esp to pathogenesis of Enterococcus faecium endocarditis. Microbes Infect. 2011, 13, 1185-1190. [CrossRef]

41. Hendrickx, A.P.; van Luit-Asbroek, M.; Schapendonk, C.M.; van Wamel, W.J.; Braat, J.C.; Wijnands, L.M.; Bonten, M.J.; Willems, R.J. SgrA, a nidogen-binding LPXTG surface adhesin implicated in biofilm formation, and EcbA, a collagen binding MSCRAMM, are two novel adhesins of hospital-acquired Enterococcus faecium. Infect. Immun. 2009, 77, 5097-5106. [CrossRef]

42. Islam, S.U. Clinical uses of probiotics. Medicine 2016, 95, e2658. [CrossRef] [PubMed]

43. Saavedra, J.M. Clinical applications of probiotic agents. Am. J. Clin. Nutr. 2001, 73, 1147S-1151S. [CrossRef] [PubMed]

44. Ljungquist, O.; Kampmann, C.; Resman, F.; Riesbeck, K.; Tham, J. Probiotics for intestinal decolonization of ESBL-producing Enterobacteriaceae; a randomized, placebo-controlled clinical trial. Clin. Microbiol. Infect. 2019. [CrossRef] [PubMed]

45. Strandwitz, P.; Kim, K.H.; Terekhova, D.; Liu, J.K.; Sharma, A.; Levering, J.; McDonald, D.; Dietrich, D.; Ramadhar, T.R.; Lekbua, A.; et al. GABA-modulating bacteria of the human gut microbiota. Nat. Microbiol. 2019, 4, 396-403. [CrossRef]

46. Donskey, C.J.; Hoyen, C.K.; Das, S.M.; Farmer, S.; Dery, M.; Bonomo, R.A. Effect of oral Bacillus coagulans administration on the density of vancomycin-resistant enterococci in the stool of colonized mice. Lett. Appl. Microbiol. 2001, 33, 84-88. [CrossRef]

47. Manley, K.J.; Fraenkel, M.B.; Mayall, B.C.; Power, D.A. Probiotic treatment of vancomycin-resistant enterococci: a randomised controlled trial. Med. J. Aust. 2007, 186, 454-457. [CrossRef]

48. Kim, Y.; Mylonakis, E. Caenorhabditis elegans immune conditioning with the probiotic bacterium Lactobacillus acidophilus strain NCFM enhances gram-positive immune responses. Infect. Immun. 2012, 80, 2500-2508. [CrossRef]

49. Duchinski, K.; Moyer, C.L.; Hager, K.; Fullerton, H. Fine-scale biogeography and the inference of ecological interactions among neutrophilic iron-oxidizing zetaproteobacteria as determined by a rule-based microbial network. Front. Microbiol. 2019, 10, 2389. [CrossRef]

50. Lebreton, F.; Manson, A.L.; Saavedra, J.T.; Straub, T.J.; Earl, A.M.; Gilmore, M.S. Tracing the Enterococci from Paleozoic Origins to the Hospital. Cell 2017, 169, 849-861. [CrossRef]

51. Coudeyras, S.; Marchandin, H.; Fajon, C.; Forestier, C. Taxonomic and strain-specific identification of the probiotic strain Lactobacillus rhamnosus 35 within the Lactobacillus casei group. Appl. Environ. Microbiol. 2008, 74, 2679-2689. [CrossRef]

52. Vidal, M.; Forestier, C.; Charbonnel, N.; Henard, S.; Rabaud, C.; Lesens, O. Probiotics and intestinal colonization by vancomycin-resistant enterococci in mice and humans. J. Clin. Microbiol. 2010, 48, 2595-2598. [CrossRef] [PubMed]

53. Zmora, N.; Zilberman-Schapira, G.; Suez, J.; Mor, U.; Dori-Bachash, M.; Bashiardes, S.; Kotler, E.; Zur, M.; Regev-Lehavi, D.; Brik, R.B.; et al. Personalized gut mucosal colonization resistance to empiric probiotics is associated with unique host and microbiome features. Cell 2018, 174, 1388-1405. [CrossRef] [PubMed]

54. De Regt, M.J.; Willems, R.J.; Hene, R.J.; Siersema, P.D.; Verhaar, H.J.; Hopmans, T.E.; Bonten, M.J. Effects of probiotics on acquisition and spread of multiresistant enterococci. Antimicrob. Agents Chemother. 2010, 54, 2801-2805. [CrossRef] [PubMed]

55. Davido, B.; Batista, R.; Michelon, H.; Lepainteur, M.; Bouchand, F.; Lepeule, R.; Salomon, J.; Vittecoq, D.; Duran, C.; Escaut, L.; et al. Is faecal microbiota transplantation an option to eradicate highly drug-resistant enteric bacteria carriage? J. Hosp. Infect. 2017, 95, 433-437. [CrossRef] [PubMed]

56. Li, X.; Song, L.; Zhu, S.; Xiao, Y.; Huang, Y.; Hua, Y.; Chu, Q.; Ren, Z. Two strains of lactobacilli effectively decrease the colonization of VRE in a mouse model. Front. Cell Infect. Microbiol. 2019, 9, 6. [CrossRef]

57. Sava, I.G.; Heikens, E.; Huebner, J. Pathogenesis and immunity in enterococcal infections. Clin. Microbiol. Infect. 2010, 16, 533-540. [CrossRef] 
58. Kreikemeyer, B.; Gamez, G.; Margarit, I.; Giard, J.C.; Hammerschmidt, S.; Hartke, A.; Podbielski, A. Genomic organization, structure, regulation and pathogenic role of pilus constituents in major pathogenic Streptococci and Enterococci. Int. J. Med. Microbiol. 2011, 301, 240-251. [CrossRef]

59. Bourgogne, A.; Hilsenbeck, S.G.; Dunny, G.M.; Murray, B.E. Comparison of OG1RF and an isogenic $f s r B$ deletion mutant by transcriptional analysis: the Fsr system of Enterococcus faecalis is more than the activator of gelatinase and serine protease. J. Bacteriol. 2006, 188, 2875-2884. [CrossRef]

60. Qin, X.; Singh, K.V.; Weinstock, G.M.; Murray, B.E. Effects of Enterococcus faecalis fsr genes on production of gelatinase and a serine protease and virulence. Infect. Immun. 2000, 68, 2579-2586. [CrossRef]

61. Millette, M.; Cornut, G.; Dupont, C.; Shareck, F.; Archambault, D.; Lacroix, M. Capacity of human nisin- and pediocin-producing lactic Acid bacteria to reduce intestinal colonization by vancomycin-resistant enterococci. Appl. Environ. Microbiol. 2008, 74, 1997-2003. [CrossRef]

62. Crouzet, L.; Derrien, M.; Cherbuy, C.; Plancade, S.; Foulon, M.; Chalin, B.; van Hylckama Vlieg, J.E.T.; Grompone, G.; Rigottier-Gois, L.; Serror, P. Lactobacillus paracasei CNCM I-3689 reduces vancomycin-resistant Enterococcus persistence and promotes Bacteroidetes resilience in the gut following antibiotic challenge. Sci. Rep. 2018, 8, 5098. [CrossRef] [PubMed]

(C) 2020 by the authors. Licensee MDPI, Basel, Switzerland. This article is an open access article distributed under the terms and conditions of the Creative Commons Attribution (CC BY) license (http://creativecommons.org/licenses/by/4.0/). 